\title{
MM-PNEMO: a mathematical model to assess handoff delay and packet loss
}

\author{
Shayla Islam ${ }^{1}$, Aisha Hassan Abdalla Hashim², Mohammad Kamrul Hasan ${ }^{3}$, Md. Abdur Razzaque \\ ${ }_{1,2}^{1}$ Department of Electrical and Computer Engineering, International Islamic University Malaysia, Malaysia \\ ${ }^{3}$ Department of Electrical and Electronics Engineering, Universiti Malaysia Sarawak, Malaysia \\ ${ }^{1,4}$ Department of Computer Science and Engineering, Green University of Bangladesh, Dhaka-1207, Bangladesh \\ ${ }^{4}$ Department of Computer Science and Engineering, University of Dhaka, Bangladesh
}

\begin{tabular}{l}
\hline \hline Article Info \\
\hline Article history: \\
Received Jan 29, 2019 \\
Revised Mar 5, 2019 \\
Accepted Mar 25, 2019
\end{tabular}

Keywords:

Handoff delay

MM-PNEMO

Multihomming

NEMO BSP

Network mobility

PNEMO

\begin{abstract}
Wireless networks incorporate Mobile Nodes (MNs) that use wireless access networks to communicate. However, the communication among these MNs are not remained stable due to the poor network coverage during inter mobility. Moreover, the wireless nodes are typically small that results in resource-constrained. Thus, it is uphill to use algorithms having giant processing power or memory footprint. Accordingly, it is essential to check schemes consistently to evaluate the performance within the probable application scenario. To do so, numerical analysis could be a notable method to grasp the performance of mobility management schemes as well as the constraint of evolving mobility management solutions specifically for multi-interfaced MR in Proxy NEMO environment. This paper analyzes handoff performance by using a mathematical model of Multihoming-based scheme to support Mobility management in Proxy NEMO (MM-PNEMO) environment. Moreover, a comparative study has been made among the standard Network Mobility Basic Support Protocol (NEMO BSP), Proxy NEMO (PNEMO) and MM-PNEMO scheme respectively. The performance metrics estimated for these schemes are mainly handoff delay and packet loss. This paper also analysed the packet loss ratio and handoff gain as a function of cell radius, number of SMR and velocity respectively. It is apparent that, the MM-PNEMO scheme shows lower packet loss ratio (1\%) compared to NEMO-BSP (11\%) and P-NEMO (6\%).
\end{abstract}

Copyright $\odot 2019$ Institute of Advanced Engineering and Science. All rights reserved.

\section{Corresponding Author:}

Shayla Islam,

Department of Electrical and Computer Engineering,

International Islamic University Malaysia,

53100 Kuala Lumpur, Malaysia.

Email: iium19612@hotmail.com; shayla.cse@gub.edu.bd

\section{INTRODUCTION}

The exponential growth of the wireless access technology includes higher capacity, extended coverage with supproting seamless inter technology handoff [1-2]. However, this inter technology handoff is becoming one of the utmost significant concern in order to provide Quality of Service (QoS) for time sensitive applications (i.e. VoIP, Video) in mobile networks [3-8]. QoS can be specified as handoff delay, packet loss, packet delivery ratio, and throughput. Investigation of these performance metrics is particularly fruitful to evaluate each mobility entities performance in mobile networks [9-10].

In mobile wireless networks, mobility models are significant building blocks for numerical-based analysis. It has a substantial impact on the performance evaluation of the mobility management schemes in NEMO [11]. In order to determine the movement rate of Mobile Router (MR) or Mobile Node (MN), it is essential to select an accurate mobility model. The most familiar mobility models utilized in mobile networks 
nemely Random Waypoint Mobility (RWM) model, City Section Mobility (CSM) model, Manhattan Mobility (MM) model as well as Fluid-flow Mobility (FM) model [11-13].

The basis of this work is to know the functioning mechanism of the mobility management schemes and to determine which protocol provide better handoff performance. The contribution of this paper includes: (i) constructing a numerical model to compare the applicability and efficiency of the MM-PNEMO scheme with that of the standard NEMO-BSP and PNEMO respectively. (ii) Investigate and analyze the handoff performance in terms of handoff delay gain and packet loss ratio.

\section{MM-PNEMO}

Explaination of the MM-PNEMO scheme as shown in Figure 1 offers the location update procedure in order to separate the new attachment of the serving MR (SMR) which is termed as 'fast registration' process from the particular flow movement which is entitled as 'flow-based routing' process [6]. As soon as the New Flow-enabled MR (NFMR) identifies the new attachment of SMR on the target network, the NFMR sends Early Proxy Binding Update (EPBU) message via Handover Initiations (HI) message to the FLMA for initiating the fast registration process without enable flow-based routing information. During fast registration mechanism, the Flow-based Local Mobility Anchor Point (FLMA) accomplishes few tasks for the new attachment such as allocating a new Home Network Prefix (HNP) and Mobile Network Prefix (MNP) of the Physical Interface 2 (PI 2), building new Binding Catch Entry (BCE) for the SMR as well as creating a new tunnel among the FNMR and the FLMA. If the NFMR identifies that the SMR is connected to the new PI (i.e. PI 2), it transmits PBU message containing FMNP option to FLMA in order to initiate flow-based routing process. Once the process is completed successfully, a PBA message is directed from the FLMA to the NFMR. However, if the network entity (i.e. CFMR, NFMR or FLMA) is not informed about the HI message with encapsulated EPBU option, the SMR will initiates PNEMO handoff process.

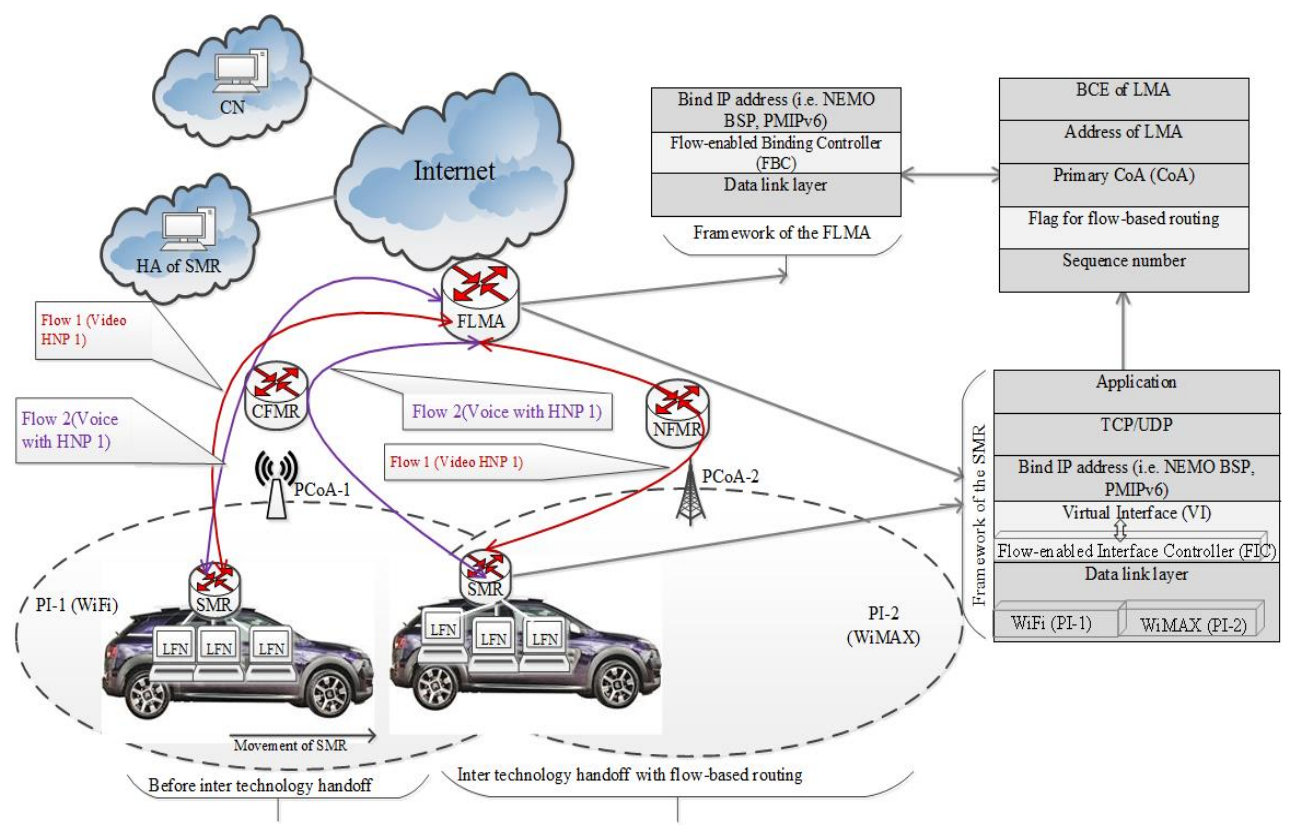

Figure 1. Basic architecture of MM-PNEMO architecture [6-7]

\section{NUMERICAL ANALYSIS}

In this section, a numerical model is developed to evaluate the performance of the MM-PNEMO scheme [6-7]. The MM-PNEMO scheme is then benchmarked with that of the NEMO-BSP and P-NEMO scheme [9-10], [14]. P-NEMO is compared with MM-PNEMO scheme since both schemes integrates firmly-entrenched PMIPv6 which is a network-based localized mobility protocol in NEMO to address mobility issues [15-17]. In contrast, NEMO-BSP is the standard protocol for any mobility support management aiming at NEMO network and it is also an improved version of the Mobile IPv6. The considered performance metrics to evaluate the performance of MM-PNEMO scheme are average handoff 
delay and its impact on packet loss. These metrics are notable since they are directly linked to the goals for the proposed model.

\subsection{Assumptions and notations}

To simply the comparison with NEMO-BSP and P-NEMO, it is assumed that the HA in NEMO-BSP is positioned in the similar place as Flow-enabled LMA (FLMA) in MM-PNEMO scheme and LMA in P-NEMO. Likewise, it is assumed that Access Routers (ARs) in NEMO-BSP are located at the identical place as MAGs in P-NEMO and FMRs in MM-PNEMO scheme. As the wired link is robust, it is assumed that the failure of message transmission is not anticipated over the wired link while the failure of message transmission is anticipated over the radio access link. Furthermore, it is also assumed that the SMR is connecting with one Corresponding Node $(\mathrm{CN})$. Moreover, Binding Update Refreshment (BUR) cost is not considered in this analysis.

\subsection{Numerical model}

The City Section Mobility (CSM) model which offers a practical movement pattern for SMRs in a town or city as depicts in Figure 2 [11]. In real world situations, cars have to follow traffic guidelines as they do not have the capability to roam without any obstacles, buildings and so on. Hence, it is essential for all SMRs to monitor predefined routes as well as behaviour procedures in this movement model. The stochastic principles of CSM model have been familiarized [18]. Therefore, the MM-PNEMO, NEMO-BSP and P-NEMO scheme adopts those principles to estimate the mobility rate as well as cell residence time.

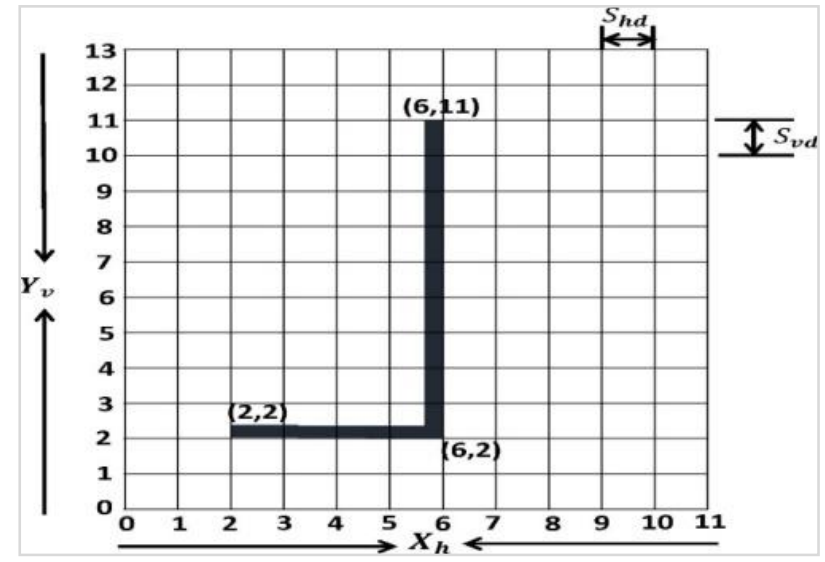

Figure 2. Structure of a road in CSM model $[7,11]$

According to CSM model, the speed limit is set for each street and the area is symbolised via a grid of lanes starting an individual sector of an urban. Each SMR begins at a defined crossing on two lanes. After that, it randomly selects an end point. Once reaching at the destination, the SMR pauses for a random time. After that, it randomly selects a new destination. Thus, the same process is repetitive and each movement is named as an epoch. The main aim of this section is to analyse the cell residence time and the mobility rate of SMR in a cell. Therefore, cell residence time of the SMR $\left(T_{S M R}\right)$ can be calculated:

$$
T_{S M R}=\frac{E(t)+E(p)}{E(c)}
$$

where, the estimated number of SMR's epoch time and pause time is represented as $E(t)$ and $E(p)$ respectively. Estimated number of SMR's cell crossings in an epoch can be symbolized as $E(c)$. It is anticipated that the moving speed of the SMR is v. Then, $E(t)$ can be expressed as:

$$
E(t)=\left(\frac{X_{h} \times\left(N_{V R}+1\right)}{3 \times N_{V R}}+\frac{Y_{v} \times\left(N_{H R}+1\right)}{3 \times N_{H R}}\right) \times \frac{1}{v}
$$


In (2), the number of horizontal and vertical road can be represented as $N_{H R}$ and $N_{V R}$ respectively whereas horizontal and vertical length of dissection area is indicated as $X_{h}$ and $Y v$ respectively. Thus, $N_{H R}$ and $N_{V R}$ can be expressed as:

$$
\begin{aligned}
& N_{H R}=\frac{X_{h}}{S_{h d}}+1 \\
& N_{V R}=\frac{Y_{v}}{S_{v d}}+1
\end{aligned}
$$

In (3) and (4), distance of horizontal and vertical road is symbolized as $S_{h d}$ and $S_{v d}$ correspondingly. Since the random pause time exists in between 0 to Umax to evade collisions at each road intersection, hence $\mathrm{E}(p)$ can be expressed as:

$$
E(p)=\frac{1}{2} \times U_{\max }
$$

It is considered that, each Access Point (AP) coverage area is circular with radius $\mathrm{r}$ and $r>S_{h d}$ and $r>S_{v d}$. It is also assumed that, $2 \times \mathrm{r}=K 1 \times S_{h d}=K 2 \times S_{v d}$. For all the movement towards horizontal and vertical direction (i.e. $K 1 \times S_{h d}$ to $2 K 1 \times S_{h d}$ ), there should be at most two APs crossings . Therefore, $E(c)$ can be represented as:

$$
\begin{aligned}
& E(c)= \\
& {\left[\frac{1}{6 \times N_{V R}^{2}}\left\{\begin{array}{l}
\left(m^{2}+m\right) \times K 1 \times \\
\left(6 N_{V R}-4 m \times K 1+K 1+3\right)
\end{array}\right\}\right]+} \\
& {\left[\frac{1}{6 \times N_{H R}^{2}}\left\{\begin{array}{l}
\left(n^{2}+n\right) \times K 2 \times \\
\left(6 N_{H R}-4 n \times K 2+K 2+3\right)
\end{array}\right\}\right]}
\end{aligned}
$$

It is noted that, the expected number of APs crossings reduces with the higher numbers of K1, K2 (i.e., 2r/Shd and 2r/Svd). Greater numbers of K1, K2 indicate larger coverage zone for APs, following in smaller number of AP crossings. On the contrary, expected number of APs crossings rises with the larger values of $N_{H R}$ and $N_{V R}$. Larger values of $N_{H R}$ and $N_{V R}$ indicate smaller coverage zone for APs, following in greater number of AP crossings.

For simplicity, it is also assumed that an access gateway area is equal to an AP area. Hence, the SMR handoff rate $(\mu \mathrm{h})$ can be estimated as follows:

$$
\mu_{h}=\frac{1}{T_{S M R}}
$$

The numerical model for performance investigation of the MM-PNEMO scheme is illustrated in Figure 3, presenting communication paths among related nodes and routers. 


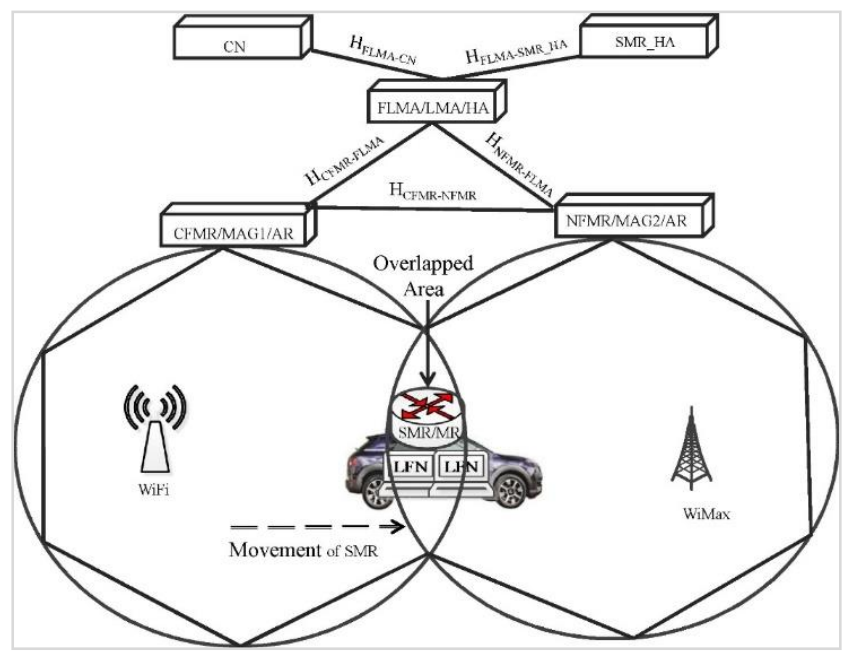

Figure 3. Numerical framework [7]

\section{RESULTS AND ANALYSIS}

The parameters are detailed in Table 1 [7-10], [19-23]. This section, the results of study is clarified and at the same time, a comprehensive discussion is also given.

Table 1. Parameters used for numerical analysis

\begin{tabular}{cc}
\hline Parameter & Value \\
\hline NSMR & $1-20$ \\
TLS & {$[50-300] \mathrm{ms}$} \\
Bwl & $54 \mathrm{Mb} / \mathrm{s}$ \\
Bwd & $1000 \mathrm{Mb} / \mathrm{s}$ \\
twl & $2 \mathrm{~ms}$ \\
twd & $.5 \mathrm{~ms}$ \\
HSMR-FMR=HMR-AR=HMR-MAG & 1 \\
HFLMA-FMR=HHA-AR=HLMA-MAG & 5 \\
HCN-FLMA=HCN-HA=HCN-LMA & 5 \\
HCFMR-NFMR=HAR-AR=HMAG1-MAG2 & 1 \\
LRS=LRA & 52 Bytes \\
LBA=LPBA & 52 Bytes \\
LBU=LPBU & 72 Bytes \\
LHI=LHAck & 52 Bytes \\
LRA & 92 Bytes \\
E(TSMR) & {$[10-100]$ second } \\
\hline
\end{tabular}

\subsection{Impact of tunnelling weight factor on handoff delay (HD)}

The Handoff Delays (HD) of MM-PNEMO scheme, NEMO-BSP and P-NEMO are investigated in Figure 4 as a function of tunneling weight factor $(\tau)$. It shows that, the delays for all schemes are increasing with changing the $\tau$. $\tau$ indicate the amount of traffic density over the link among the FMRs in the proposed scheme. When road traffic is high, the number of SMRs moving between the same FMRs increase. Therefore, $\tau$ can imply road traffic characteristics. Since, the delay over wireless link rises, the handoff delay rises in MM-PNEMO scheme (182.8 millisecond) and P-NEMO (543.1 millisecond). However, these delays are not critical as in NEMO-BSP (1034 millisecond). From the observation in Figure 4, it is confirmed that the MM-PNEMO scheme and P-NEMO show frequent handoff compared to NEMO-BSP. This is because, the LU over the wireless link is avoided and no tunnelled packets are conveyed over the wireless link. Moreover, the DAD mechanism in NEMO-BSP counts for a huge portion of handoff delay. Hence, it is essential to reduce this DAD delay to improve handoff performance. Subsequently, MM-PNEMO also outperforms P-NEMO by taking the advantage of previous knowledge about the network conditions and its flows during flow-enabled fast registration phase. 


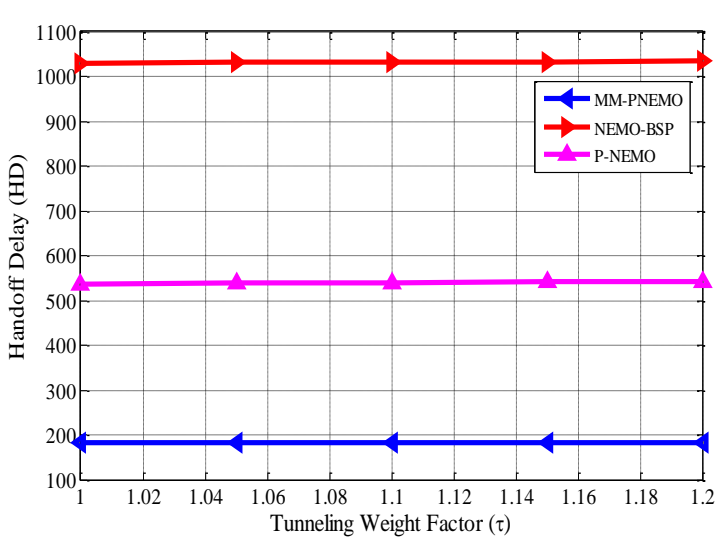

Figure 4. Handoff delay vs tunnelling weight factor [7]

\subsection{Impact of tunneling weight factor on HD gain}

The influence of tunneling weight factor $(\tau)$ on handoff delay gain is illustrated in Figure 5 . It is noticed that, the overall gain of P-NEMO decreases as $\tau$ increases, whereas that of MM-PNEMO remains almost the same, regardless of the increasing $\tau$. This is due to the tunnelling burden of P-NEMO that effects in disruption of session continuity. Accordingly, P-NEMO and MM-PNEMO outperform NEMO-BSP. Therefore, it can be summed up from Figure 5 that, the MM-PNEMO scheme can vastly improve the handoff performance compared to NEMO-BSP and P-NEMO regardless of increasing $\tau$. This is because, SMR is capable to split the application flow burden among multiple access technology during inter technology handoff.

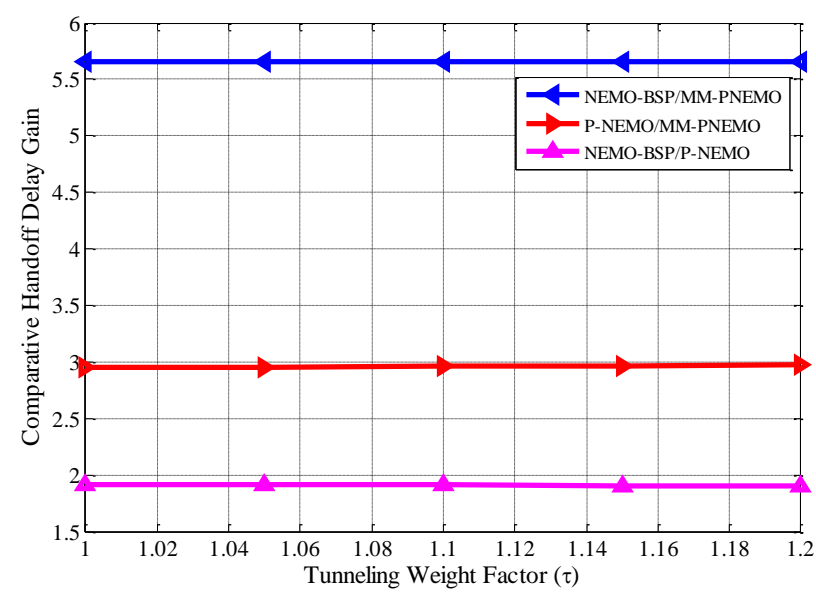

Figure 5. Handoff delay gain vs tunneling weight factor

\subsection{Impact of number of SMR on packet loss (PL)}

In Figure 6, the amount of packet loss during inters technology handoff for each scheme is depicted for varying the number of SMRs and different cell residence time (TSMR). It is observed from Figure 5 that, the total packet loss of each scheme increases for changing the number of SMRs with setting lower residence times (i.e. TSMR=20 sec). If the TSMR is varying from 20 to $100 \mathrm{sec}$, the SMR is most likely to stay in a cell and rarely moves to another position. Thus, the packet loss during handoff is very small as depicts in Figure 5. Basically, packet loss is proportional to the handoff disruption time. Therefore, the cases of the handoff mode for MM-PNEMO shows lower packet loss compared to P-NEMO and NEMO-BSP. 


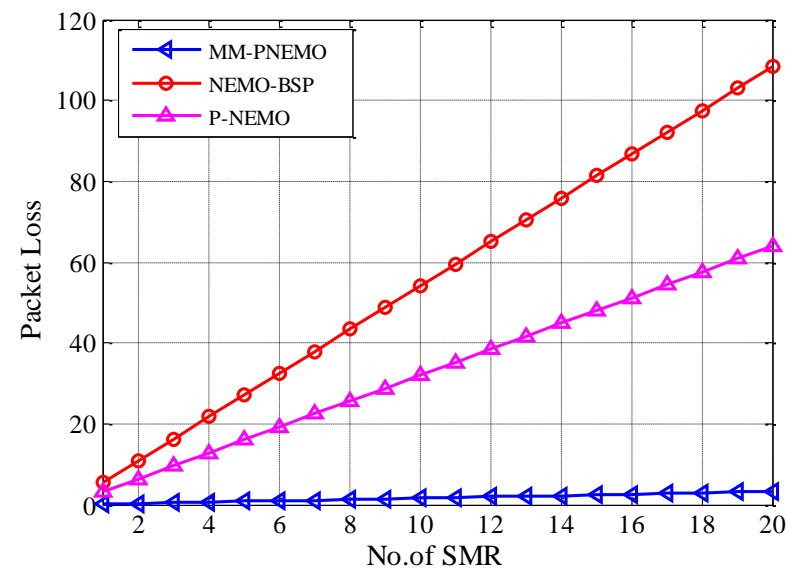

Figure 6. Packet loss vs number of SMR (TSMR=80sec) [7]

\subsection{Impact of mobility rate and average session length on packet loss (PL)}

In Figures 7 and 8, the amount of packet loss for each scheme is depicted for varying mobility rate $(\mu \mathrm{h})$ and different session length $(\lambda \mathrm{s})$. Typically, total packet loss decreases with lower $\mu \mathrm{h}$ of SMR. When $\mu \mathrm{h}$ increases, the SMR moves frequently and changes subnet recurrently because of its high mobility. Thus, includes a number of SMR handoffs which leads to increase packet loss as shown in Figure 7.

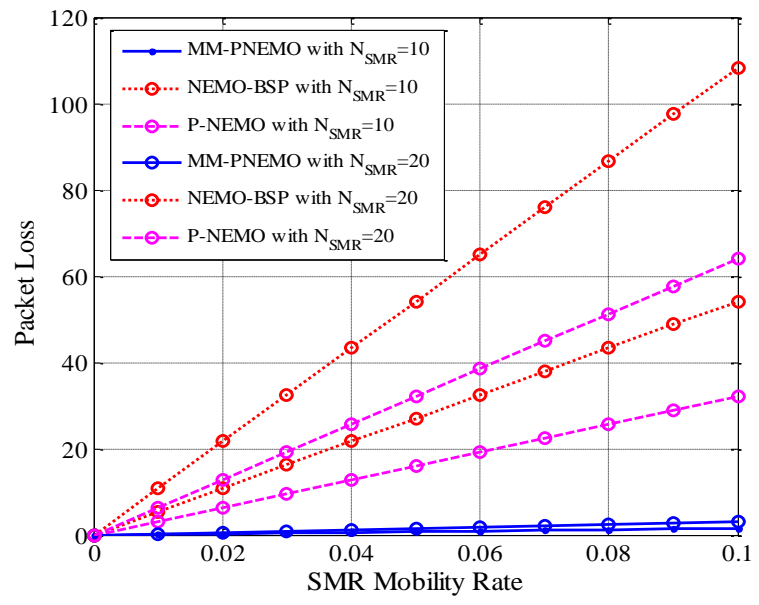

Figure 7. Packet loss vs SMR mobility rate [7]

Correspondingly, the packet loss is proportional to $\lambda \mathrm{s}$. In Figure 8, it is apparent that packet loss increases proportionally with the $\lambda$ s for all schemes. Network-based localized schemes (i.e., MM-PNEMO and P-NEMO) outperform NEMO-BSP. This is because due to the elimination of mobility signalling over the wireless link (when $\lambda$ s increases). Besides, the proposed MM-PNEMO consumes less packet loss compared to NEMO-BSP and P-NEMO due to the support of inter technology handoff in P-NEMO. This means that MM-PNEMO is better suited for real time applications where periodic packets are sent at higher rates. 


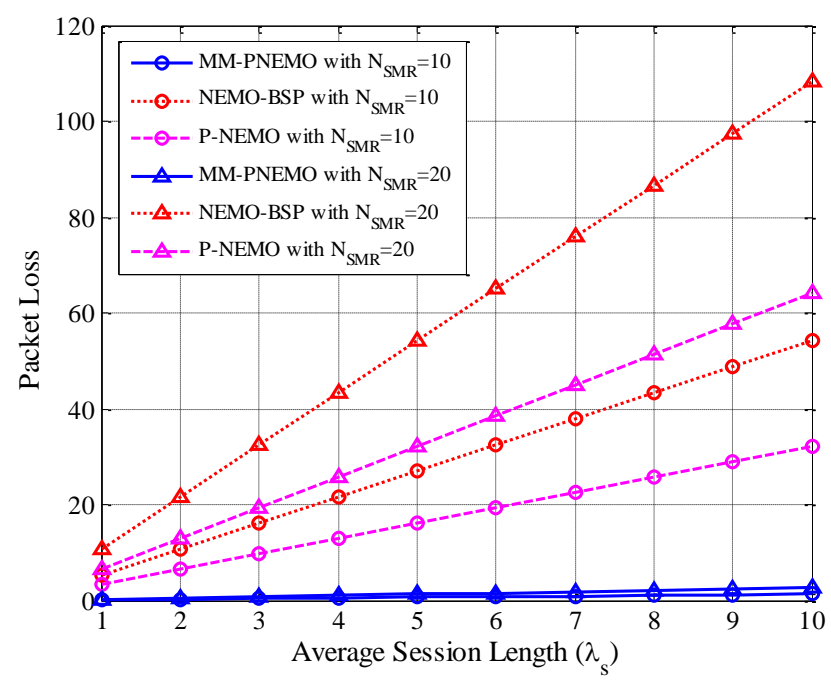

Figure 8. Packet loss vs average session length (packets/flow) [7]

\subsection{Impact of radius, no. of SMR and speed on packet loss ratio}

Figure 9 to 11 illustrate packet loss ratio as a function of cell radius, number of SMR and velocity respectively. In Figure 10 and 11, the the number of SMR and cell radius is varied; whereas the speed and the residence time are kept constant (i.e. speed $=60$ meter/second and $\mathrm{T}_{\mathrm{SMR}}=20$ second). It is apparent from both figures that, the packet loss ratio fluctuates among $1 \%$ to $12 \%$. Correspondingly, the moving speed is set from 1 to 80 meter/second (like that of usual moving bus or car) as depicts in Figure 11. It is apparent from Figures 9 to 11 that, the packet loss ratio increases with changing the velocity of the SMR. This is because, higher velocity results in smaller residence time which leads to increase number of handoffs. As a result, MM-PNEMO and P-NEMO scheme shows lower packet loss ratio compared to NEMO-BSP. In contrast, MM-PNEMO shows lower packet loss ratio (1\%) compared to NEMO-BSP (11\%) and P-NEMO (6\%) as shown in Figure 9.

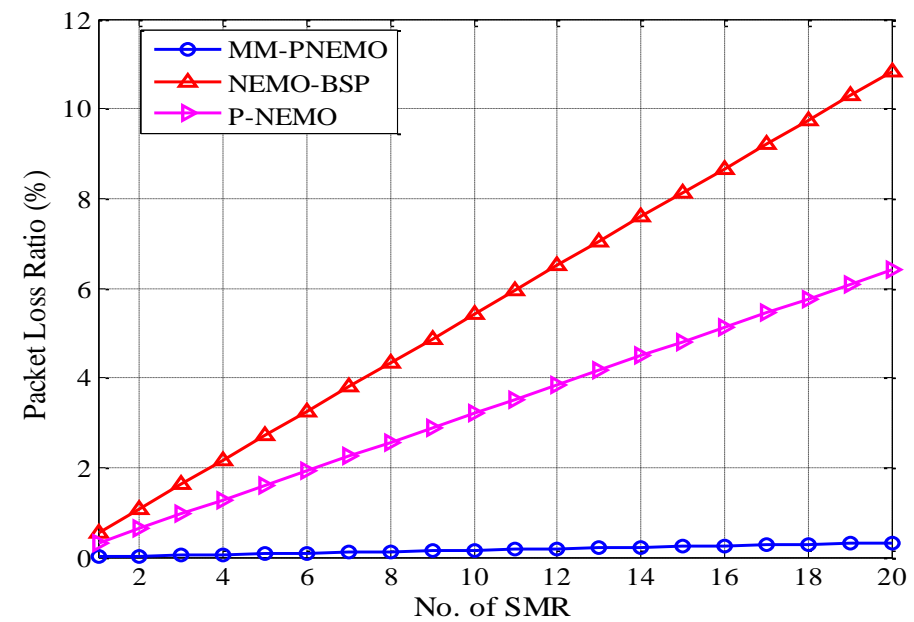

Figure 9. Packet loss ratio vs number of SMR $(\mathrm{TSMR}=20 \mathrm{sec})$ 


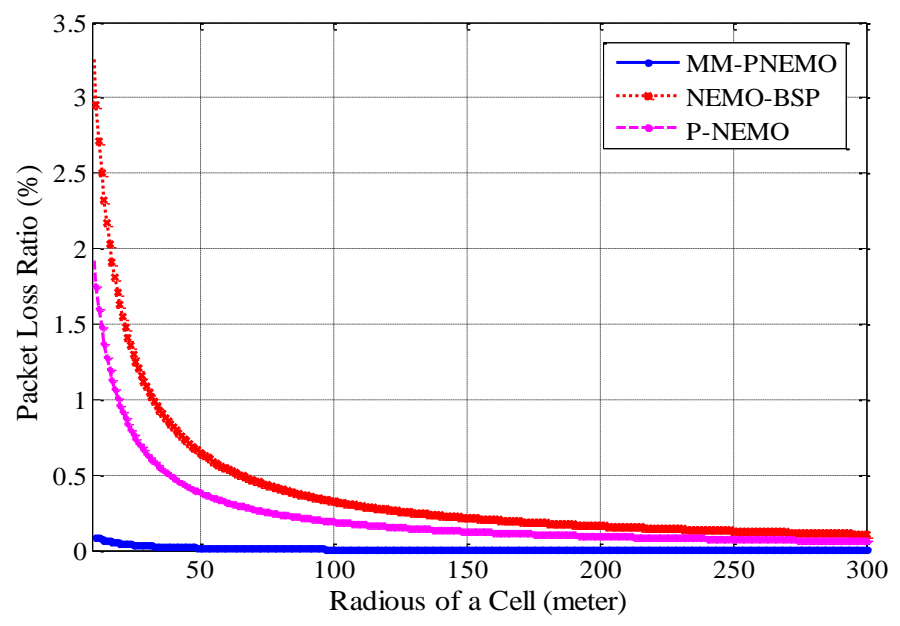

Figure 10. Packet loss ratio vs cell radius $\left(\mathrm{T}_{\mathrm{SMR}}=20 \mathrm{sec}\right)$

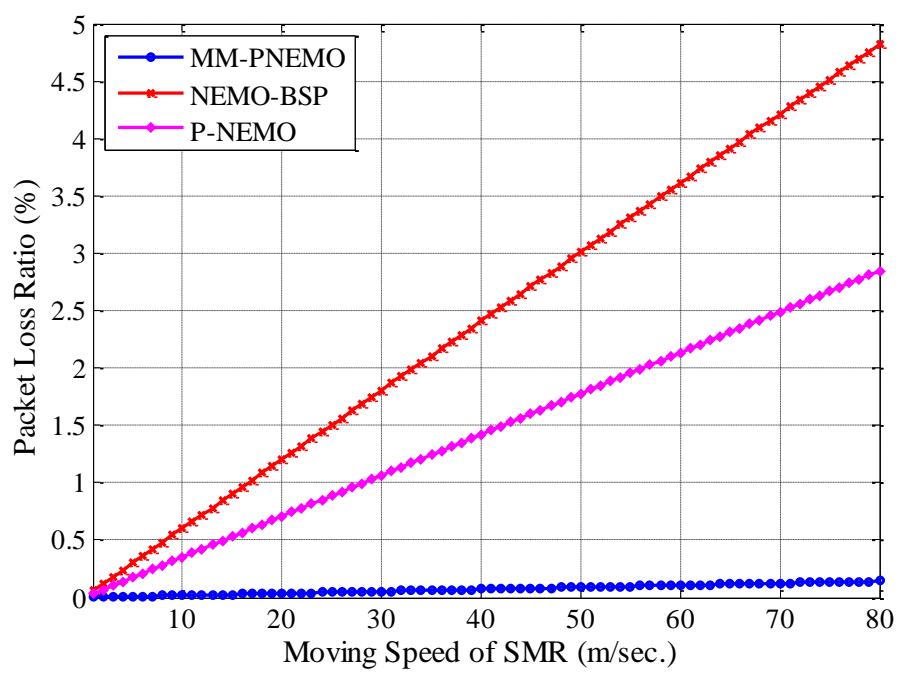

Figure 11. Packet loss ratio vs speed $(\mathrm{TSMR}=60 \mathrm{sec})$

\section{CONCLUSION}

NEMO-BSP is the basic standard protocol that is enahanced from the MIPV6. However, the study suggests that the NEMO-BSP take over the llimitations of MIPV6 especially for ineffective routing path, single point of failure, higher handoff delays as well as high packet loss. Hence, to cope handoff issues, this paper evaluates handoff performance by using a mathematical model of Multihoming-based scheme to support Mobility management in Proxy NEMO (MM-PNEMO) environment. As of the study, the MM-PNEMO is paralleled with the standard NEMO-BSP and P-NEMO for benchmarking. The mathematical results demostrated that, the MM-PNEMO significantly maximizes the packet delivery ratio upto $99 \%$ in compare with NEMO BSP and P-NEMO respectively.

\section{ACKNOWLEDGEMENTS}

A special thanks to the Research Management Centre (RMC), International Islamic University Malaysia (IIUM) for the grant.

\section{REFERENCES}

[1] Hasan, M. K., Saeed, R. A., Hashim, A. H. A., Islam, S., Alsaqour, R. A., \& Alahdal, T. A.," Femtocell network time synchronization protocols and schemes". Research Journal of Applied Sciences, Engineering and Technology, vol. 4, no. 23, pp. 5136-5143, 2012. 
[2] M. K. Hasan et al., "Inter-cell interference coordination in LTE-A HetNets: A survey on self organizing approaches," 2013 International Conference on Computing, Electrical And Electronic Engineering (ICCEEE), Khartoum, 2013, pp. 196-201.

[3] Hashim, A. H. A., Hassan, W. H., Islam, S., Saeed, R. A., Hasan, M. K., Daoud, J. I., \& Khalifa, O. O., "An enhanced macro mobility management scheme in NEMO environment to achieve seamless handoff," World Applied Sciences Journal, vol. 21, pp. 35-39, 2013.

[4] Devarapalli, V., Wakikawa, R., Petrescu, A., \& Thubert, P., "Network mobility (NEMO) basic support protocol", IETF RFC 3963, 2005.

[5] Lee, C.-W., M.C. Chen, and Y.S. Sun, "A novel network mobility management scheme supporting seamless handover for high-speed trains," Computer Communications, vol. 37, pp. 53-63, 2014.

[6] Islam, S., Abdalla, A. H., \& Hasan, M. K., "Novel multihoming-based flow mobility scheme for proxy NEMO environment: A numerical approach to analyse handoff performance". SCIENCEASIA, vol. 43, pp.27-34, 2017.

[7] Islam, S., Hashi, A. H. A., Razzaque, A., \& Hasan, M. K., "A Numerical Model to Analyze Handoff Delay and Packet Loss in PNEMO Environment". In 2018 7th International Conference on Computer and Communication Engineering (ICCCE), 2018, pp. pp. 130-134.

[8] Islam, S., Hashim, A. H. A., Habaebi, M. H., \& Hasan, M. K., "Design and implementation of a multihoming-based scheme to support mobility management in NEMO". Wireless Personal Communications, vol. 95, no. 2, pp. 457-473, 2017.

[9] J. Lee and T. Ernst, "Lightweight NEtwork MObility Within PMIPv6 for Transportation Systems," in IEEE Systems Journal, vol. 5, no. 3, pp. 352-361, Sept. 2011.

[10] J. Lee, T. Ernst and N. Chilamkurti, "Performance Analysis of PMIPv6-Based NEtwork MObility for Intelligent Transportation Systems," in IEEE Transactions on Vehicular Technology, vol. 61, no. 1, pp. 74-85, Jan. 2012.

[11] Hossain, M. S., \& Atiquzzaman, M., "Cost analysis of mobility protocols," Telecommunication Systems, vol. 52, no. 4, pp. 2271-2285, 2013.

[12] M. S. Hossain, M. Atiquzzaman and W. Ivancic, "Performance evaluation of multihomed NEMO," 2012 IEEE International Conference on Communications (ICC), Ottawa, ON, 2012, pp. 5429-5433.

[13] M. S. Hossain, M. Atiquzzaman and W. Ivancic, "Performance comparison between multihomed network mobility protocols," 2012 IEEE Global Communications Conference (GLOBECOM), Anaheim, CA, 2012, pp. 5260-5265.

[14] S. Ryu, J. Choi and K. Park, "Performance evaluation of improved fast PMIPv6-based network mobility for intelligent transportation systems," in Journal of Communications and Networks, vol. 15, no. 2, pp. 142-152, April 2013.

[15] Calderon, M., Bernardos, C., \& Soto, I., "PMIPv6 and Network Mobility Problem Statement". IETF, <draftbernardos-netext-pmipv6-nemo-ps-02>, 2012.

[16] Choi, J.-I., W.-K. Seo, and Y.-Z. Cho, "Efficient network mobility support scheme for proxy mobile IPv6". EURASIP Journal on Wireless Communications and Networking, vol. 2015, no. 210, pp. 1-13, 2015.

[17] R. Kuntz, J. Montavont and T. Noel, "Multihoming in IPv6 mobile networks: progress, challenges, and solutions," in IEEE Communications Magazine, vol. 51, no. 1, pp. 128-135, January 2013.

[18] M. S. Hossain and M. Atiquzzaman, "Stochastic Properties and Application of City Section Mobility Model," GLOBECOM 2009 - 2009 IEEE Global Telecommunications Conference, Honolulu, HI, 2009, pp. 1-6.

[19] C. Makaya and S. Pierre, "An Analytical Framework for Performance Evaluation of IPv6-Based mobility Management Protocols," in IEEE Transactions on Wireless Communications, vol. 7, no. 3, pp. 972-983, March 2008.

[20] Islam, Shayla, Aisha Hassan Abdalla, Mohamed Hadi Habaebi, Wan Haslina Hassan, and Mohammad Kamrul Hasan. "Design and Simulation of a Multihoming-based Inter-system Handoff Scheme in NEMO." Elektronika ir Elektrotechnika 22, no. 1 (2016): 92-96.

[21] Choi, Hyon-Young, et al. "Design and Simulation of a Flow Mobility Scheme Based on Proxy Mobile IPv6." JIPS 8.4 (2012): 603-620.

[22] Melia, Telemaco, et al. "IP flow mobility in PMIPv6 based networks: solution design and experimental evaluation." Wireless Personal Communications 61.4 (2011): 603-627.

[23] Musa, Abdulwaheed, Saad Osman Bashir, and Aisha Hassan Abdalla. "Review and Assessment of Electromagnetic Wave Propagation in Sand and Dust Storms at Microwave and Millimeter Wave Bands---Part I." Progress In Electromagnetics Research 40 (2014): 91-100.

\section{BIOGRAPHIES OF AUTHORS}

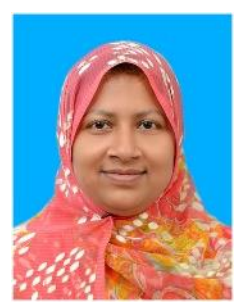

Currently, Dr. Shayla Islam is appointed as a post-doctoral fellow in International Islamic University Malaysia (IIUM). In addition, she is working as an assistant professor in Green University of Bangladesh (on leave). She has completed PhD degree in Engineering from Electrical and Computer Engineering (ECE) department at International Islamic University Malaysia (IIUM) in 2016 under Malaysian International Scholarship (MIS). She completed her MSc. at the department of Electrical and Computer Engineering in International Islamic University Malaysia in 2012. She completed her BSc. in Computer Science and Engineering from International Islamic University Chittagong, Bangladesh. Dr. Shayla has awarded a Silver medal for her research work at International Islamic University Malaysia. In consequences, she has also awarded a Young Scientist Award for the 
contribution of research paper at 2nd International Conference on Green Computing and Engineering Technologies, 2016 (ICGCET'16), Organized by the Department of Energy Technology, Aalborg University, Esbjerg, Denmark. Previously, she was appointed as a lecturer in Green University of Bangladesh (GUB) from 2007 to 2009. Her current research interests include data communications and networking, computer networks and wireless communication, network mobility in heterogeneous network, computer architecture \&cloud computing. She published more than 50 papers in international journals and conferences (WoS/ISI/ESCI and Scopus indexed). She works as a reviewer for many international conferences as well as journals.

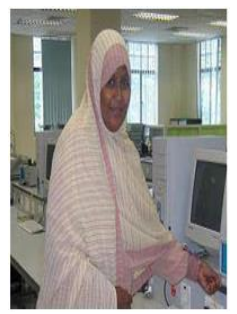

Aisha-Hassan Abdalla Hashim is currently a Professor at the Department of Electrical and Computer Engineering, Faculty of Engineering, International Islamic University Malaysia. She completed his bachelor degree studies in Electrical Engineering at University of Gezira, Sudan. She completed her MSc and PhD in Electrical and Computer Engineering from International Islamic University Malaysia. Her research interests include Wireless Networking, Network Mobility, and Cognitive Radio. She is a registered senior member of the IEEE (SMIEEE) and member of the Institution of Institution of Engineering and Technology (MIET) Professor Aisha has been serving the engineering society through practicing engineering, technical seminars, workshop, teaching, publications, volunteer works and patent. She is actively participating in curriculum development and program accreditation. She is a member of the Department Board of Studies for several years. She received Best Teacher Award by IIUM during the Quality Day in 2007. She has been appointed as external examiner/visiting professor/adjunct professor at different universities. Professor Aisha is actively involved in research and postgraduate programs. She has published more than 250 journal/conference papers. Professor Aisha is actively involved in research and postgraduate programs. She has acquired several research grants from IIUM, Ministry of Higher Education (MOHE) and Ministry of Science, Technology and Innovation (MOSTI). She is the author of five engineering books. Dr. Aisha has been supervising at least forty masters and $\mathrm{PhD}$ students.

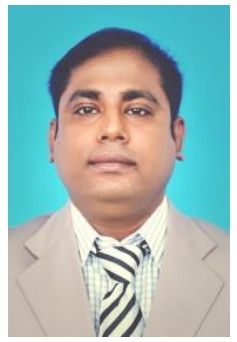

Mohammad Kamrul Hasan is currently a postdoctoral researcher at the department of Electrical and Computer Engineering in International Islamic University, Malaysia. He has completed his $\mathrm{Ph} . \mathrm{D}$. in Electrical and Computer Engineering, in the year of 2016, from the department of Electrical and Computer Engineering, International Islamic University, Malaysia. He was a research fellow in the same university during June 2016-June 2017. He received his Masters degree in Communication Engineering from International Islamic University, Malaysia in 2012. His current research interests include Smart Grid Computing, Wireless Communication, Network Mobility, Cognitive Network, and Artificial Intelligence. He published several number of international indexed journals and conferences. $\mathrm{He}$ is a Senior Member of Institute of Electrical and Electronics Engineers (SMIEEE), Member of Institution of Engineering and Technology (MIET), and Internet Society (ISOC). Dr. Mohammad is an editor panel member of FENG Research Bulletin in Universiti Malaysia Sarawak.

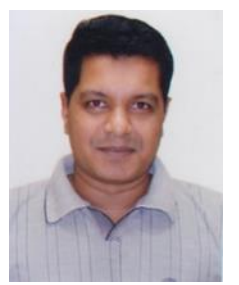

Md. Abdur Razzaque received his B.S. and M.S. degrees from the University of Dhaka, Bangladesh in 1997 and 1999, respectively. He obtained his $\mathrm{PhD}$ degree in Wireless Networking from the Department of the Computer Engineering, School of Electronics and Information, Kyung Hee University, South Korea in 2009. He was a research professor in the same university during 20102011. He worked as a visiting scholar in Stratford University, USA in 2017. Currently, Dr. Razzaque is working as a Professor and Chairperson at the Department of Computer Science and Engineering, Green University of Bangladesh (GUB). Professor Razzaque is actively contributing to the engineering society as a group leader of Green Networking Research Group (http://cse.du.ac.bd/gnr) at the Department of Computer Science and Engineering, University of Dhaka, Bangladesh. His research interest is in the area of modeling, analysis and optimization of wireless networking protocols and architectures, wireless sensor networks, body sensor networks, cooperative communications, sensor data clouds, internet of things, cognitive radio networks, and smart grid communications. He has published several number of research papers in international conferences and in indexed journals. Dr. Razzaque is an editor of Journal of Network and Computer Applications (JNCA), associate editor of IEEE Access, TPC chair of ICIET 2018, General Co-chair of IEEE R10HTC 2017, TPC member of IEEE HPCC 2013-2018, SKIMA 2016-2018, ICOIN 2010-2018, NSysS 2015-2018. He is a senior member of IEEE, member of IEEE Communications Society, IEEE Computer Society, Internet Society (ISOC), Pacific Telecommunications Council (PTC) and KIPS. 